\title{
Lasing Action in Single Subwavelength Particles Supporting Supercavity Modes
}

\author{
Vasilii Mylnikov, Son Tung Ha, Zhenying Pan, Vytautas Valuckas, Ramón Paniagua-Domínguez, \\ Hilmi Volkan Demir, and Arseniy I. Kuznetsov*
}

Cite This: ACS Nano 2020, 14, 7338-7346

Read Online

ACCESS | Lلll Metrics \& More | 国 Article Recommendations | (1) Supporting Information

ABSTRACT: On-chip light sources are critical for the realization of fully integrated photonic circuitry. So far, semiconductor miniaturized lasers have been mainly limited to sizes on the order of a few microns. Further reduction of sizes is challenging fundamentally due to the associated radiative losses. While using plasmonic metals helps to reduce radiative losses and sizes, they also introduce Ohmic losses hindering real improvements. In this work, we show that, making use of quasibound states in the continuum, or supercavity modes, we circumvent these fundamental issues and realize one of the smallest purely semiconductor nanolasers thus far. Here, the nanolaser structure is based on a single

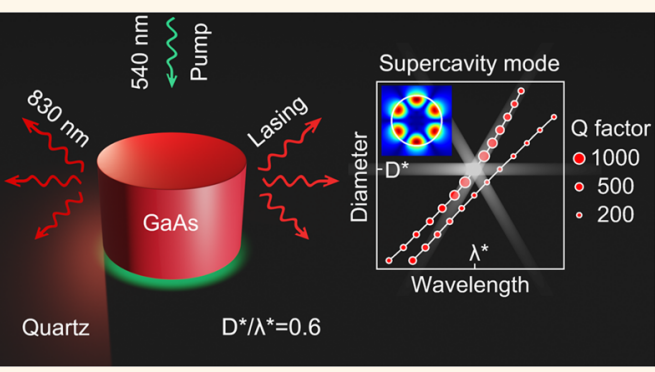
semiconductor nanocylinder that intentionally takes advantage of the destructive interference between two supported optical modes, namely Fabry-Perot and Mie modes, to obtain a significant enhancement in the quality factor of the cavity. We experimentally demonstrate the concept and obtain optically pumped lasing action using GaAs at cryogenic temperatures. The optimal nanocylinder size is as small as $500 \mathrm{~nm}$ in diameter and only $330 \mathrm{~nm}$ in height with a lasing wavelength around $825 \mathrm{~nm}$, corresponding to a size-to-wavelength ratio as low as 0.6 .

KEYWORDS: dielectric nanoantennas, laser, nanolaser, bound state in the continuum, Mie resonance, gallium arsenide

I $\mathrm{n}$ the past decade, resonant dielectric nanoantennas have emerged as a promising platform for nanophotonic applications. Low Ohmic losses and CMOS compatibility, accompanying small form factors, are the key advantages of these systems that make them particularly attractive for industrial applications as compared to prior designs based on plasmonic metals. ${ }^{1}$ In recent years, moreover, significant efforts are being made in combining dielectric nanoantenna concepts with active material platforms ${ }^{2}$ to extend this promising approach toward efficient directional light sources. By now, the use of dielectric nanoantennas in emission processes has been mainly focused on enhancing fluorescence from molecules, ${ }^{3-8}$ enhancing the Raman signal ${ }^{9-11}$ and enhancing photoluminescence (PL) from the nanoantenna material itself. ${ }^{12-15}$ Due to the limited quality factors ( $Q$ factors) of low-order modes excited in dielectric nanoantennas, lasing has not been demonstrated yet for particle sizes significantly smaller than the wavelength, with the recent exceptions of micrometer-sized systems in which subwavelength nanoantennas are arranged in lattices. ${ }^{16,17}$ Hence, besides strongly dissipative systems involving metals, ${ }^{2,18}$ on-chip lasers have been mostly restricted to spatially extended systems with sizes on the order of a few microns.

The smallest fully dielectric lasers were experimentally demonstrated using suspended disk structures. ${ }^{19,20}$ High-order
Mie-type modes (i.e., whispering gallery modes) were used in those designs to achieve lasing. In this regard, the smallest nanolaser reported consisted of a disk on top of a pedestal with a diameter of $627 \mathrm{~nm}$, operating in the fourth-order Mie resonance and emitting at a wavelength of $870 \mathrm{~nm}$, which corresponds to a size/wavelength ratio $\zeta=0.72 .{ }^{20}$ Very recently, one of the smallest, fully dielectric, single-particlebased laser has been demonstrated using perovskites in a nanocube configuration. ${ }^{21}$ This design also uses the fourthorder Mie-type mode and has a slightly higher size/wavelength ratio of $\zeta=0.79$ but operates at room temperature. Further reduction of size (i.e., in disk diameter) is challenging for two different reasons. First, using smaller resonators while keeping the same operating wavelength implies necessarily the use of lower order modes. Those present higher radiative losses, which leads to a drop in the $Q$ factor. Second, smaller particles have larger surface-to-volume ratios, which increases the effect

Received: April 1, 2020

Accepted: May 27, 2020

Published: May 27, 2020 

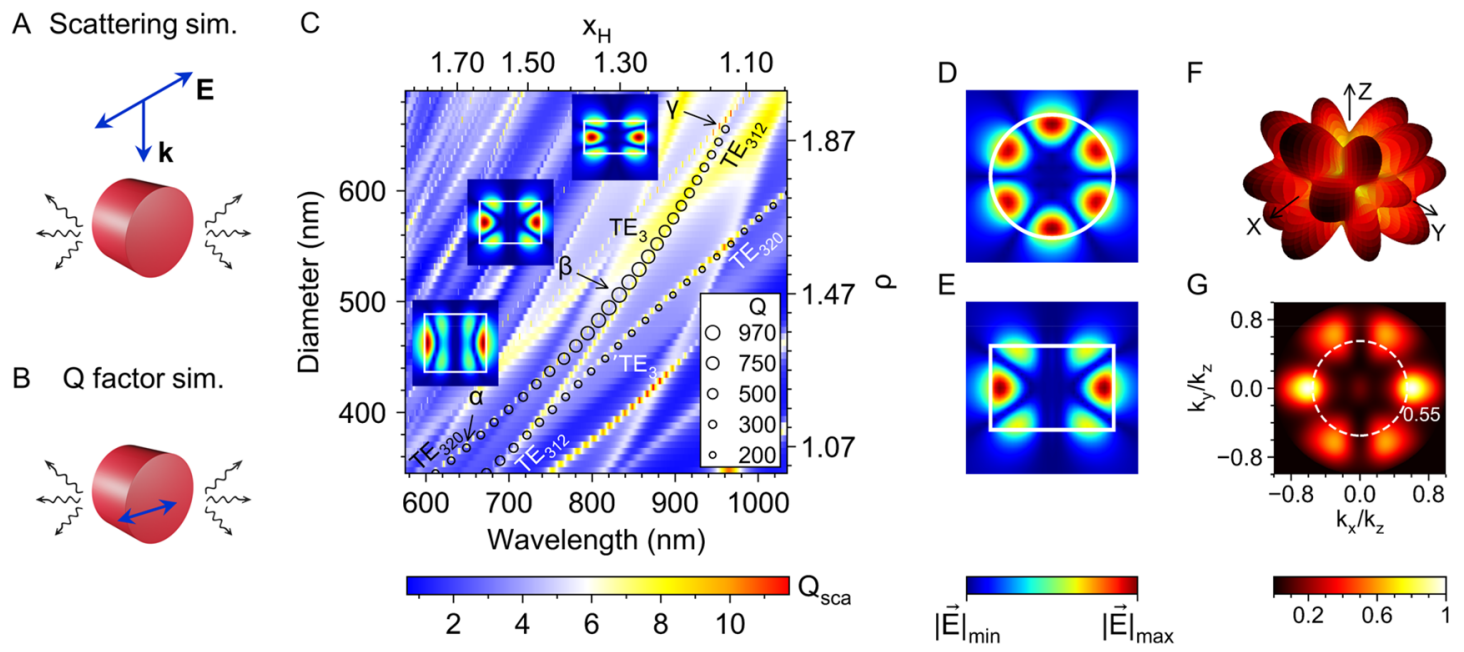

Figure 1. Simulated characteristics of a single GaAs nanocylinder $(\varepsilon=13)$ in vacuum. (A, B) Illustration of the dielectric cylinder in vacuum during scattering simulations (A) and $Q$ factor (near-field, far-field) simulations (B). (C) Scattering efficiency map $Q_{\text {sca }}$ as a function of the dimensionless parameters $x_{\mathrm{H}}=\pi H / \lambda$ (the size parameter) and $\rho=D / H$ (the cylinder aspect ratio). The alternative $x$-and $y$-axis correspond to the wavelength and diameter obtained when the height of the cylinder is fixed at $H=345 \mathrm{~nm}$. The peak positions and the $Q$ factors of the modes, obtained from the $Q$ factor simulations, are shown by black circles, their area being directly proportional to the value of the $Q$ factor. The results of the $Q$ factor simulations are shown for the quasi-TE modes of azimuthal order $i=3$ (labeled $\mathrm{TE}_{3}$ and 'TE ' $_{3}$. Only these two modes are displayed in order to highlight the anticrossing of the modes $\mathrm{TE}_{320}$ and $\mathrm{TE}_{312}$ and $Q$ factor evolution as a function of the diameter and wavelength (the $Q$ factor reaches its maximum value for the optimal parameters indicated by a point $\beta$ which is the supercavity mode point). The insets show the electric near-field maps in the $Y Z$ plane passing through the center of the cylinder for different regimes of the mode $\mathrm{TE}_{3}$. (D, E) Simulated near fields of the supercavity mode in the $X Y(\mathrm{D})$ and $Y Z(\mathrm{E})$ planes of the cylinder, respectively. White lines in (D, E) show the cylinder boundaries. (F) Simulated 3D far-field radiation pattern corresponding to the point $\beta$. (G) $2 \mathrm{D}$ projection of the simulated far-field radiation pattern corresponding to the point $\beta$. The white dashed circle shows the range of wave-vectors collected by a 0.55 NA objective (similar to the one used to collect the emission in the lasing experiment).

of surface recombination and roughness, resulting in higher losses that make the gain-loss compensation even more challenging. While the second is more difficult to overcome, without improving the fabrication processes and/or including some surface passivation methods, the first still allows some improvement from the design perspective. Thus, different approaches need to be implemented for improving the quality of the cavities in order to achieve lasing in smaller resonators.

A recent promising approach to boost the $Q$ factor of a system is engineering it to support a special type of resonant mode called bound state in the continuum (BIC). BICs are nonradiative states, thus perfectly spatially confined, which nevertheless coexist with the continuum of radiative states (i.e., despite the presence of available radiation channels). ${ }^{22}$ BICs are a general wave phenomenon, first reported in electronic systems and subsequently extended to photonic ones, ${ }^{22}$ and are true eigenmodes of the system (in fact, often referred to as embedded eigenstates). Strictly speaking, for any system comprising regular materials with $\varepsilon \neq 0, \mu \neq 0, \varepsilon \neq \pm \infty$, or $\mu \neq \pm \infty$, BICs can only be supported if the system is infinite, and their $Q$ factors are infinite. ${ }^{22}$ Parameters of a finite system, however, can be tuned close to the condition of BICs in the corresponding infinite system. ${ }^{23}$ The obtained quasiBICs are often referred to in the literature as supercavity modes and possess finite but high $Q$ factors. ${ }^{23}$ This makes supercavity modes or quasi-BICs useful for many photonics applications, ${ }^{22,24}$ including, for example, sensing ${ }^{25,26}$ and highharmonic generation. ${ }^{27-31}$

Regarding the use of BICs for lasing, one of the first quasiBIC lasers was demonstrated in 1985. It supported a symmetry-protected quasi-BIC by suppressing radiation into the transverse directions in a $1 \mathrm{D}$ distributed feedback laser cavity. $^{32}$ After that, similar quasi-BIC principles were demonstrated in many photonic crystal surface-emitting laser devices. $^{22}$ In recent years, quasi-BIC-based lasing results have been shown for on-chip systems based on nanoparticle arrays. $^{16,17}$ Going beyond the BIC supported by spatially extended systems toward single particle configurations, it should be mentioned that a spatially confined system can only support a true BIC if it contains some region in which the permittivity tends to zero. ${ }^{33,34}$ Nevertheless, several recent reports have theoretically predicted that it should, still, be possible to obtain, if not a true BIC, but a quasi-BIC or a supercavity mode in such a simple system as a single dielectric nanocylinder. ${ }^{28,35,36}$ This quasi-BIC has a Friedrich-Wintgen nature and allows some degree of radiation to escape the system. Thus, by reciprocity, it is also possible to excite it by external sources. Recent experimental studies have, indeed, demonstrated the excitation of these quasi-BICs in a single cylinder at $\mathrm{GHz}^{37}$ and optical ${ }^{30}$ frequencies. However, no experimental results of lasing in supercavity regime for a single semiconductor nanoparticle have been shown so far. In this work, elaborating on the BIC concept, we report single dielectric nanoparticle laser working in supercavity regime. This also enables the realization of fully dielectric nanolaser operating in the third azimuthal order mode for a particle on a substrate, with size/wavelength ratio as low as $\zeta=0.6$.

\section{RESULTS AND DISCUSSION}

We designed our cylindrical nanoresonator to support a quasiBIC that originates from the strong interaction of two resonant modes: a Mie-like mode (which strongly depends on the diameter of the cylinder and is largely insensitive to its height) and a Fabry-Perot (FP)-like mode (which is formed by the 
multiple reflections of the guided mode supported by the cylinder at its top and bottom interfaces and therefore highly sensitive to the cylinder height). ${ }^{35}$ The strong interaction between these two resonant modes manifests itself as an avoided resonance crossing in the scattering spectrum of the cylinder. ${ }^{37}$ Along with the avoided crossing, one of the modes exhibits line width narrowing (increase in the $Q$ factor) and a decrease in the scattering efficiency in the anticrossing region. Specifically, the quasi-BIC (the supercavity mode point) ${ }^{35}$ corresponds to geometrical parameters of the system where the maximum $Q$ factor (minimum radiation losses) is reached while the formation of the dark mode, which refers to the minimal scattering, occurs at a different point (the dark mode point). ${ }^{37}$ These two points match exactly for the case of an ideal BIC. ${ }^{37}$ Thus, there are three main signatures of the emergence of a supercavity mode of this type: anticrossing between Mie-like and FP-like modes, increase in the $Q$ factor for one of the modes, and decrease in its scattering efficiency in the anticrossing region. The use of a supercavity mode reduces the radiative losses of the system (increasing its $Q$ factor several times with respect to a regular Mie mode of the same order) $)^{35}$ enabling gain/loss compensation in nanoparticles of a smaller size.

We focus our attention on obtaining lasing from a supercavity mode of azimuthal order $i=3$. In particular, we study a single GaAs cylinder standing on a quartz substrate. Due to the relatively low gain of GaAs at room temperature, which is further reduced by the large surface recombination present in small particles, lasing experiments are performed at the liquid nitrogen temperature (i.e., $77 \mathrm{~K}$ ) to increase the quantum yield of GaAs (note that no surface passivation or any chemical treatment is applied to the nanostructures). When tuning the temperature from 300 to $77 \mathrm{~K}$, the emission peak of GaAs shifts from 870 to $830 \mathrm{~nm} .{ }^{16}$ Thus, in this work, the geometrical parameters of the cylinder (diameter $D$ and height $H)$ are optimized to achieve high $Q$ factors at $\lambda=830 \mathrm{~nm}$.

To illustrate the physical mechanism behind the supercavity mode formation in a simple way, let us first consider a cylinder with a constant relative permittivity $\varepsilon=13$ (corresponding, approximately, to GaAs at $830 \mathrm{~nm}$ wavelength and $77 \mathrm{~K}$ ) in vacuum. We perform two types of three-dimensional electromagnetic simulations-scattering simulations and $Q$ factor calculations (illustrated in parts A and B, respectively, of Figure $1)$. For the scattering simulations we study illumination of the cylinder by a transverse electric (TE) wave. The TE wave is defined as the one having incident magnetic field polarized along the axis of the cylinder and the electric field and incident wave vector perpendicular to it as shown in Figure 1A. In the $Q$ factor simulations, we excite TE modes by a pointlike dipole highlighted in Figure 1B (see the Methods for more details). We compute the scattering efficiency and the eigenmodes supported by this cylinder as a function of its geometrical parameters using full wave simulations based on the finitedifference time-domain method (see the Methods for the detailed description). The results are shown in Figure 1C. There, we plot the scattering efficiency as a function of the dimensionless parameters $x_{\mathrm{H}}=\pi H / \lambda$ (i.e., the size parameter, where the height of the cylinder is used as a characteristic dimension) and $\rho=D / H$ (the cylinder aspect ratio). Overlaid with the scattering map, we plot the result of the $Q$ factor simulations: quasi-TE eigenmodes (quasi-normal modes) corresponding to the azimuthal order $i=3$ are shown by a set of black circles, their area being directly proportional to the
$Q$ factors of the modes. Here, we use a standard mode notation $\mathrm{TE}_{i j}$, where indices $i, j$, and $l$ are the azimuthal, radial, and axial indices of the mode, respectively. "TE" or "TM" denotes the dominant polarization of the mode. ${ }^{37}$ As seen in the plot, two modes of the third azimuthal order coexist in the range of size parameters studied (denoted as $\mathrm{TE}_{320}$ and $\mathrm{TE}_{312}$ ). $\mathrm{TE}_{320}$ is the Mie-like mode, and $\mathrm{TE}_{312}$ is the FP-like mode. More important is the clear indication of their strong coupling, evidenced through an avoided spectral crossing. In the anticrossing region, the modes are hybrid; thus, the use of the standard notation might be misleading when addressing the modes in that region. To get around that, we use a simplified notation to denote the branches (modes) $\mathrm{TE}_{3}$ (higher frequency branch) and ${ }^{\prime} \mathrm{TE}_{3}$ (lower frequency branch) that originate from strong coupling of the modes $\mathrm{TE}_{320}$ and $\mathrm{TE}_{312}$. Along with avoided spectral crossing, a dramatic increase in the $Q$ factor, leading to a maximum (at the supercavity mode point denoted as $\beta$ in Figure $1 \mathrm{C}$ ) is observed for the mode $\mathrm{TE}_{3}$ near the anticrossing region, indicating that a supercavity mode has been formed. The reduction of the scattering efficiency at slightly higher diameters, with respect to the supercavity mode point of the mode $\mathrm{TE}_{3}$, is less noticeable due to relatively low permittivity. ${ }^{35}$ The optimum values of dimensionless parameters for this third azimuthal order mode are $\mathrm{x}_{\mathrm{H}} \sim 1.30$ and $\rho$ $\sim 1.47$ (the supercavity mode point). For an operational wavelength $\lambda=830 \mathrm{~nm}$, these correspond to a cylinder with height $H \sim 344 \mathrm{~nm}$ and diameter $D \sim 504 \mathrm{~nm}$. The corresponding $Q$ factor reaches a maximum value of $Q=970$. It should be noted that it has been theoretically shown ${ }^{35}$ that an infinite permittivity is formally equivalent to a spatially infinite system in which an ideal BIC may exist. Practically, this means that the higher the permittivity is, the closer to an ideal BIC the supercavity mode is. This explains why in the case of moderate permittivity of GaAs, the supercavity formation leads to a somehow moderate 5-fold increase in the $Q$ factor. This, nevertheless, turns out to be crucial to reduce the lasing threshold for the third azimuthal order mode, as will be shown in the experiment below.

To complete the characterization of the supercavity mode, the near fields and the far-field radiation pattern in the supercavity regime are depicted in Figures $1 \mathrm{D}-\mathrm{G}$. From the near-field maps (Figures $1 \mathrm{D}, \mathrm{E}$ ), the $\mathrm{TE}_{312}$ character of the mode becomes apparent. On the other hand, the far-field pattern (Figure 1F) shows that, along with the regular in-plane lobes, which are common for pure Mie modes, out-of-plane lobes are present as well. This can be explained by the fact that supercavity mode is a hybrid FP-Mie mode and, thus, it cannot be identified as a pure FP mode or a pure Mie mode. A detailed analysis of the evolution of the near-field distribution around the supercavity regime confirms that the mode $\mathrm{TE}_{3}$ has indeed three different regimes, evolving from a Mie-like one (Figure 1C at point $\alpha$ ) to the Supercavity one (Figure $1 \mathrm{C}$ at point $\beta$ ) and finally to a Fabry-Perot-like one (Figure $1 \mathrm{C}$ at point $\gamma$ ). Note that in optical experiments we collect the very edge of the emission side lobes of the supercavity mode, as expected from the simulated radiation pattern in Figure $1 \mathrm{G}$.

To experimentally demonstrate lasing for the analyzed thirdorder supercavity mode, GaAs nanocylinders standing on a quartz substrate are fabricated using the method reported in ref 16. First, a GaAs film grown on top of a commercially available GaAs wafer with a sacrificial layer of AlAs is transferred to a quartz substrate using the technique known as epitaxial lift$\mathrm{off}^{38}$ followed by a direct bonding process developed in-house 

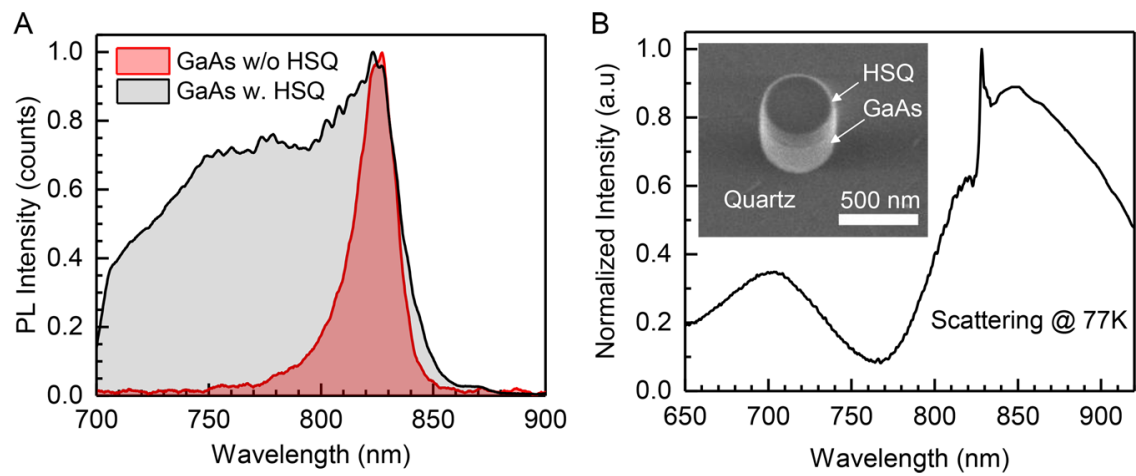

Figure 2. (A) Normalized photoluminescence spectrum measured from GaAs film without HSQ (red) and with $150 \mathrm{~nm}$ layer of HSQ on top (black). (B) Normalized experimental dark-field scattering spectrum of a single GaAs nanocylinder with parameters $D=500 \mathrm{~nm}$ and $H=$ $330 \mathrm{~nm}$. Inset: exemplary SEM image of the fabricated nanostructure.

A

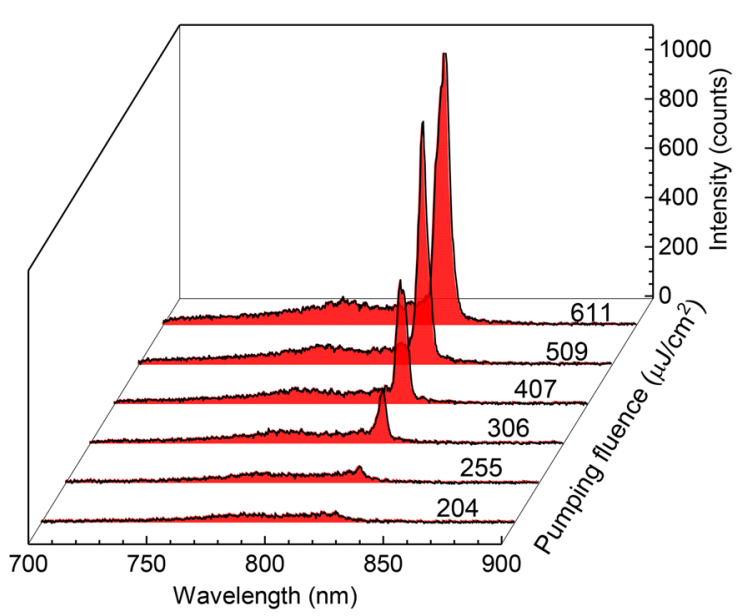

D

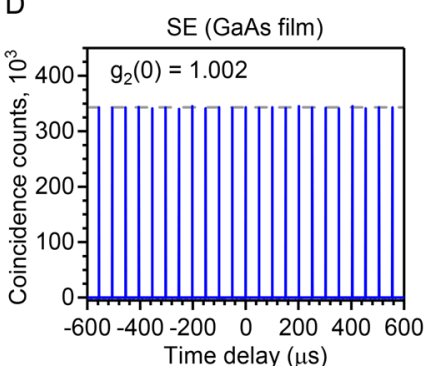

E

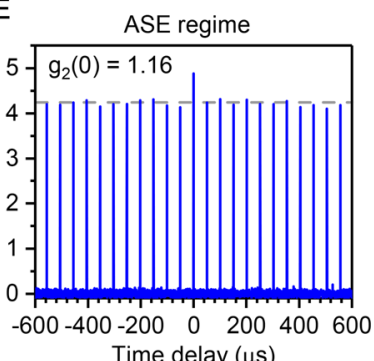

B
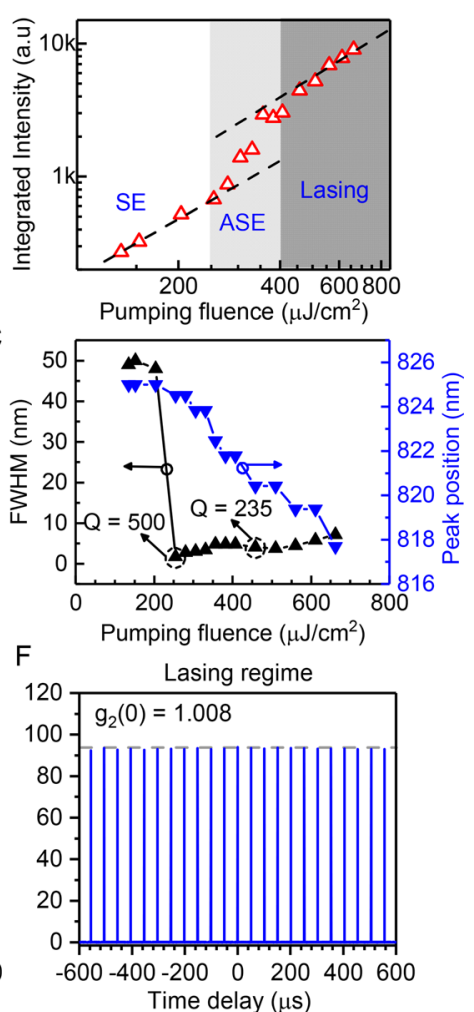

Figure 3. Lasing action in a GaAs nanocylinder. (A) Evolution of the emission spectrum of the GaAs nanocylinder with diameter $D=500$ and height $H=330 \mathrm{~nm}$ at different pumping fluences. (B) Log-log plot of output emission intensity versus pumping laser fluence showing the transitions from spontaneous emission (SE) to amplified spontaneous emission (ASE) and finally to lasing. (C) Peak position and fwhm of the lasing peak extracted from the emission spectra. (D) Intensity autocorrelation histogram for $50 \mu \mathrm{m} \times 50 \mu \mathrm{m}$ GaAs square at the pumping laser fluence $F=764 \mu \mathrm{J} / \mathrm{cm}^{2}$ and for the single GaAs nanocylinder of diameter $500 \mathrm{~nm}$ and height $330 \mathrm{~nm}$ for the pumping fluences (E) $F=382 \mu \mathrm{J} / \mathrm{cm}^{2}$ and (F) $F=433 \mu \mathrm{J} / \mathrm{cm}^{2}$.

using a wafer bonding system. The transferred film is then etched to a desired thickness using inductively coupled plasma (ICP) method. The nanocylinder pattern is then defined using a standard e-beam lithography with hydrogen silsesquioxane (HSQ) as a negative resist. The final structure is formed by an additional dry-etching step (see the Methods for a more detailed information). The SEM image of one of the fabricated nanostructures is shown in the inset of Figure 2B. Note that, despite their low index, the presence of the quartz substrate and the remaining capping HSQ layer affects the spectral position and the $Q$ factor of the supercavity mode. Thus, we reoptimize the parameters of the cylinder to account for them, obtaining a maximum $Q$ factor of 280 for optimum values of the dimensionless parameters $x_{\mathrm{H}} \sim 1.29$ and $\rho \sim 1.47$, corresponding to the cylinder height $H \sim 340 \mathrm{~nm}$ and diameter $D \sim 500 \mathrm{~nm}$ at $\lambda=830 \mathrm{~nm}$ (the complete analysis of the modes of the reoptimized geometry will be presented in details later in the text). The height of the GaAs cylinders obtained in the fabricated samples is close to the targeted one, around $330 \mathrm{~nm}$ (measured using reflectometer and tilted SEM images). The sample is then cooled down to $77 \mathrm{~K}$ using liquid nitrogen for further optical characterization (see the Methods for details). 
Before characterizing the fabricated nanostructures, we first investigate the absorption and PL spectra of GaAs films with the thickness of $350 \mathrm{~nm}$. The absorption spectrum, recorded at $77 \mathrm{~K}$, is presented in Figure S1. The spectrum clearly shows the absorption edge at $\sim 827 \mathrm{~nm}$, in good correspondence with values reported in literature for GaAs at this temperature. ${ }^{16}$ The narrow absorption peak at $\sim 830 \mathrm{~nm}$ can be attributed to the excitonic resonance of the material. For the spontaneous emission spectrum, large square areas (of $50 \mu \mathrm{m} \times 50 \mu \mathrm{m}$ ) patterned in the film using EBL, are analyzed. We do so to characterize, together, the emission of GaAs and that of HSQ resist, used to pattern the nanostructures in our experiments and known to have non-negligible fluorescence when treated with e-beam and high temperatures. ${ }^{39,40}$ Indeed, for GaAs film with HSQ resist on top, the measured photoluminescence (Figure 2A) has a broad emission spectrum from 700 to 850 $\mathrm{nm}$ (a long pass filter is used to cut the pump at $700 \mathrm{~nm}$ ), beyond that for GaAs alone which has a relatively narrow PL spectrum from 800 to $850 \mathrm{~nm}$. The above-mentioned broad spectrum is attributed to the photoluminescence of GaAs combined with that of silicon nanocrystals formed in the silica matrix of cured HSQ (whose PL can range from 650 to 800 $\mathrm{nm}),{ }^{40}$ which, in our case, most probably arises due to the high energy electron beam exposure, in a chemical reaction analogous to that happening during thermal annealing. ${ }^{41}$ Thus, in our experiments, the photoluminescence of both $\mathrm{Si}$ nanocrystals in cured $\mathrm{HSQ}^{42}$ and GaAs itself can provide gain for our nanolasers with different resonance wavelengths.

With this information in mind, we now move to the optical characterization of the nanocylinders at cryogenic temperatures, first studying their scattering spectra, to identify the formation of the supercavity mode, and then their emission under optical pumping. Figure 2B shows the characteristic dark-field scattering spectrum of a single GaAs nanocylinder with $D=500 \mathrm{~nm}$ and $H=330 \mathrm{~nm}$ (as measured using SEM). From our theoretical analysis, we expect the cylinder to support the supercavity mode at around $830 \mathrm{~nm}$ for these dimensions. Indeed, one can observe the emergence of a very narrow peak in the experimental spectrum at $\sim 828 \mathrm{~nm}$, in very good agreement with what is expected from the simulations, thus confirming the presence of the quasi-BIC mode. To demonstrate lasing action, we optically pump the nanocylinders using a femtosecond laser (with a wavelength of $530 \mathrm{~nm}, 200 \mathrm{fs}$ pulse duration and $20 \mathrm{kHz}$ repetition rate) and collect the PL using a microspectrometer setup (see detailed information in the Methods). Nanocylinders have been fabricated with a $20 \mu \mathrm{m}$ spacing, and the pumping laser spot size is $\sim 5 \mu \mathrm{m}$ in diameter, ensuring that each cylinder is individually irradiated during optical measurements. Figure $3 \mathrm{~A}$ shows the PL spectrum evolution of the GaAs nanocylinder with diameter $D=500 \mathrm{~nm}$ and height $H=330 \mathrm{~nm}$, supporting the supercavity mode, when pumped under different laser fluences. It can be seen that when the pumping fluence is larger than $260 \mu \mathrm{J} / \mathrm{cm}^{2}$, a pronounced, narrow peak appears at $\sim 825$ $\mathrm{nm}$. By plotting the integrated emission intensity versus the pumping fluence in log-log scale (Figure 3B) one can clearly see the "S" shape that indicates the transition from spontaneous emission (SE) to amplified spontaneous emission (ASE) and finally to lasing, with a lasing threshold of $\sim 300 \mu \mathrm{J} /$ $\mathrm{cm}^{2}$. The PL peak position and full-width-at-half-maximum (fwhm) as a function of the pumping fluence, extracted from the spectra, are shown in Figure 3C. Based on the fwhm, we observe a maximum $Q$ factor of 235 in the lasing regime (in good agreement with the theoretically calculated value of 280 , as discussed above). From the analysis of the lasing peak position, a clear blue-shift is observed when the pumping fluence is increased, which can be attributed to the BursteinMoss effect associated with the band-filling. ${ }^{43,44}$ The lasing peak broadening that can be also observed when increasing the fluence can be attributed to two effects: band-filling and thermal broadening. In our case, we observe the blue-shift of the lasing peak when increasing the pumping fluence, which is a strong evidence of the band-filling effect. Given the high pumping fluence (few hundreds $\mu \mathrm{J} / \mathrm{cm}^{2}$ ) and the high repetition rate $(20 \mathrm{kHz})$ of the femtosecond laser, in addition to the relatively bad thermal-dissipation setup (single GaAs nanopillar on quartz) used in our lasing experiment, we believe that thermal broadening effect also contributes to the broadening of the lasing peak. This explains the drop of the $Q$ factor from 500 to 235 as shown in Figure 3C.

To further confirm the coherent nature of the lasing emission, autocorrelation measurements using a HanburyBrown-Twiss setup ${ }^{16,45}$ are performed. Parts D-F of Figure 3 show coincidence counts at different time delays for three different regimes: SE (Figure 3D), ASE (Figure 3E), and lasing (Figure $3 \mathrm{~F}$ ). The normalized second-order correlation function at zero time delay $g_{2}(0)$ is defined as the ratio between coincidence count number of the pulse at zero time delay and an averaged count number over 22 other neighboring pulses. It is noted that the autocorrelation measurement for SE (Figure $3 \mathrm{D}$ ) is performed on relatively large patterned GaAs squares (of $50 \mu \mathrm{m} \times 50 \mu \mathrm{m}$ ). This is to ensure that an adequate amount of signal can be collected and analyzed. It, however, does not change the nature of the SE from the materials composing the nanolaser. Due to their larger size, the photoluminescence intensity of these squares is several times greater than that of the single GaAs nanocylinder used to measure $g_{2}(0)$ in the ASE and lasing regimes, which explains why the coincidence count number in Figure $3 \mathrm{D}$ is substantially higher than those of Figures 3E,F. The $g_{2}(0)$ for the SE case is calculated to be 1.002 , which is significantly smaller than the theoretical value for an ideal thermal light source (i.e., $\mathrm{g}_{2}(0)=2$ ). This is because the coherence time of the $\mathrm{SE}$ is much lower than the detection limit of our experimental setup $(\sim 81 \mathrm{ps})$, in accordance with similar observations reported in earlier studies. ${ }^{16,46}$ Nevertheless, we are able to measure a clear decrease in $g_{2}(0)$ upon transitioning between the ASE and lasing regimes, as shown in Figure 3E,F, with the $g_{2}(0)$ reducing from 1.16 (for ASE regime, $F=382$ $\mu \mathrm{J} / \mathrm{cm}^{2}$ ) to 1.008 (for lasing regime, $F=433 \mu \mathrm{J} / \mathrm{cm}^{2}$ ) and, thus, clearly indicating the coherent behavior of the nanolaser.

We now prove that lasing is indeed obtained for the third azimuthal order supercavity mode by studying how a slight detuning of the geometrical parameters from the optimal ones affects the performance of the system. For that, lasing and dark-field scattering measurements are carried out for cylinders of the same height, namely $H=330 \mathrm{~nm}$, but with diameters above and below the optimum one. In particular, Figure $4 \mathrm{~A}$ shows the dark-field scattering spectra of GaAs cylinders with diameter ranging from 420 to $653 \mathrm{~nm}$ with an increment of $\sim 5$ $\mathrm{nm}$. The mode highlighted by green dashed line in Figure $4 \mathrm{~A}$ corresponds to the third azimuthal order mode $\mathrm{TE}_{3}$ from the simulation (see Figure 4B). Higher and lower order modes can also be seen from the scattering data in Figure 4A. For a better visualization of the formation of the supercavity mode, the scattering spectra for several selected radii around the 

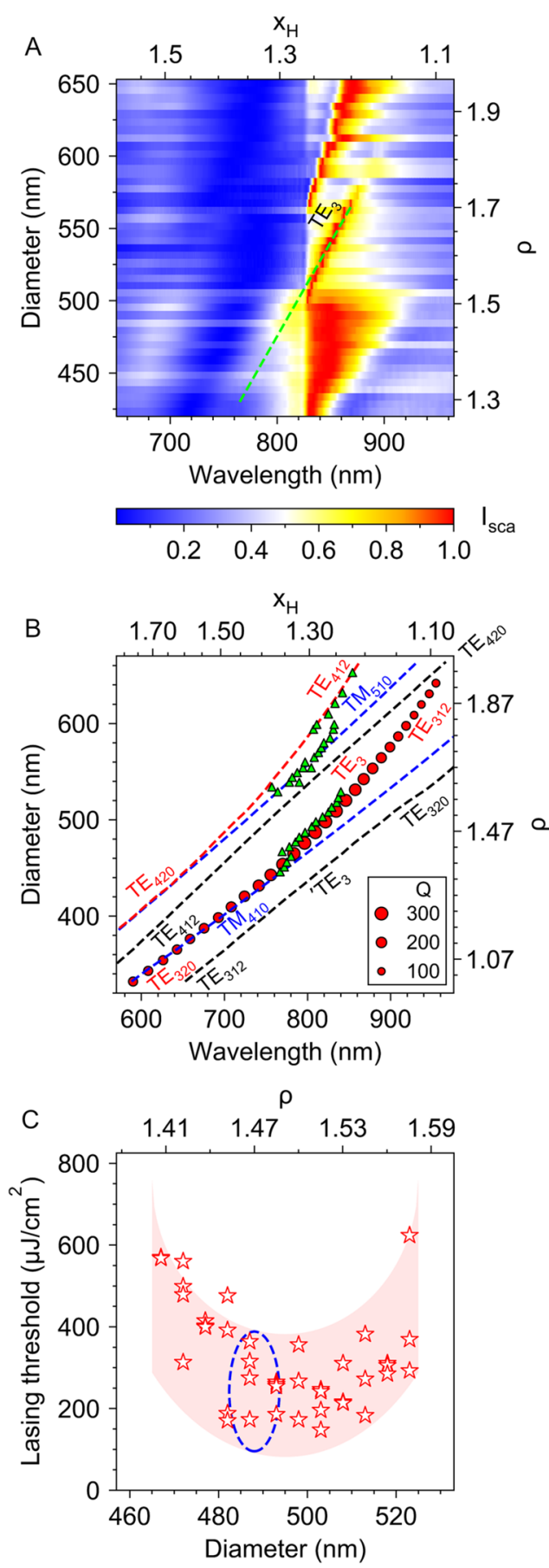

Figure 4. Optical characteristics of the mode $\mathrm{TE}_{3}$ in the vicinity of the supercavity regime. (A) Normalized experimental dark-field scattering spectra of GaAs nanocylinders with fixed height $H=330$ $\mathrm{nm}$ and varying diameter $D=420-653 \mathrm{~nm}$ with an increment of 5 $\mathrm{nm}$. The green dashed line is a guide to eyes indicating the mode $\mathrm{TE}_{3}$. (B) Theoretically obtained peak positions of the modes supported by a single dielectric nanocylinder $\varepsilon=13$ on a substrate, retrieved from the $Q$ factor simulations, shown in the region of interest of dimensionless parameters $x_{\mathrm{H}}, \rho$. The alternative $x$ - and $y$-axis correspond to the wavelength and diameter obtained when the height of the cylinder is fixed at $H=332 \mathrm{~nm}$. The modes denoted as $\mathrm{TE}_{320}$ and $\mathrm{TE}_{312}$ are strongly coupled which is manifested by the anticrossing. At optimal parameters these modes destructively interfere in the far field which leads to the formation of the supercavity regime for the mode $\mathrm{TE}_{3}$ for which the corresponding $Q$ factors are indicated by red circles (their area being directly proportional to the value of the $Q$ factor). Strong
Figure 4. continued

coupling of the modes $\mathrm{TE}_{420}$ and $\mathrm{TE}_{412}$ manifests itself as an avoided resonance crossing. Modes $\mathrm{TM}_{410}$ and $\mathrm{TM}_{510}$ are regular Mie modes. The green triangles show the experimentally obtained lasing peak positions for cylinders with fixed height $H=330 \mathrm{~nm}$ and different diameters. (C) Measured lasing threshold values (depicted as red stars) shown as a function of the diameter of the nanocylinder for the mode $\mathrm{TE}_{3}$. The red shadowed area is a guide to eyes. The vicinity of the optimal diameter value is highlighted by the blue dashed line (where the mode $\mathrm{TE}_{3}$ undergoes the supercavity regime in theory, $\rho \sim 1.47)$. This has a slight discrepancy with experimental results, where the minimal threshold is obtained at $\rho \sim 1.51$.

optimum one are depicted in Figure S2A. It should be noted that, due to the presence of high absorption at wavelengths below the excitonic resonance, it is not possible to observe the full evolution of the scattering peak associated with the formation of the supercavity mode (from broad to narrow and back to broad) but only the narrowing of the resonance. Thus, we explore an alternative experimental method, relying on the analysis of lasing threshold, to prove that the third azimuthal order TE mode used for lasing indeed undergoes the formation of the supercavity regime, as explained below.

Figure 4B shows the theoretically obtained (using $Q$ factor simulations) peak positions of the quasi-normal eigenmodes in the span of parameters $\lambda$ and $D$ of our interest. In the case of the mode $\mathrm{TE}_{3}$, the corresponding $Q$ factors are also plotted by red circles, so that the supercavity regime can be readily identified. Superimposed in the same plot, the green triangles depict the experimental lasing peak positions obtained for various disk diameters. As can be seen, there is a good agreement between the simulated peak positions and the lasing peaks observed in the experiment. The discrepancy between theory and experiment is minimal and lays within the error of diameters determined by SEM and the possible slight deviations in the material parameters. Importantly, we can achieve lasing for the third azimuthal order mode $\mathrm{TE}_{3}$ for a relatively wide range of cylinder diameters due to the low divergence of quality factor near the optimal value. This gives us flexibility to tune the emission wavelength of the nanolaser within the gain spectrum of the active materials involved (note that, in our case, these refer not only to GaAs, but also to the Si nanocrystals obtained inside HSQ resist after the e-beam exposure). Besides the mode $\mathrm{TE}_{3}$, lasing is also obtained for bigger nanocylinders supporting higher azimuthal order modes (up to fifth-order $\mathrm{TM}_{510}$ mode as shown in Figure $4 \mathrm{~B}$ ). It is important to note that, for nanocylinders with diameters close to the optimum $(D \sim 500 \mathrm{~nm})$, the third-order mode of interest $\left(\mathrm{TE}_{3}\right)$ and the fourth-order Mie mode $\left(\mathrm{TM}_{410}\right)$ are spectrally close $(\sim 20 \mathrm{~nm}$ away from each other, according to the $Q$ factor simulations, as can be seen in Figure 4B). Thus, it is crucial to observe both the third- and fourth-order modes in the lasing experiments to unequivocally prove that the mode $\mathrm{TE}_{3}$ is identified correctly. The detailed view of the emission spectra contributed by both these modes simultaneously is shown in Figure S2B. Moreover, as can be seen in Figure 4B, the lasing peak positions for all modes (third, fourth, and higher azimuthal orders) match well with the theory, further supporting the correct identification of the mode $\mathrm{TE}_{3}$. Figure $\mathrm{S} 3$ shows additional information on the modes $\mathrm{TE}_{3}$ and $\mathrm{TM}_{410}$ obtained from the simulations. The overlap of the near-field 
maxima (Figures S3B-E) with the sidewalls of the cylinder is more pronounced for the mode $\mathrm{TM}_{410}$ compared to that of the mode $\mathrm{TE}_{3}$. Thus, resonances of higher azimuthal orders are more sensitive to the sidewall roughness created during the dry-etch process, which tends to lower the $Q$ factor in experiments. As a consequence, compared to mode $\mathrm{TE}_{410}$, mode $\mathrm{TE}_{3}$ near the supercavity regime seems to be more robust against fabrication imperfections and therefore more suitable to obtain lasing in practical devices.

As mentioned above, in order to demonstrate the formation of the supercavity regime of the mode, the lasing threshold is measured and analyzed for several nanocylinders with different diameters around the optimum, as shown in Figure 4C. For each diameter, two to four similar nanocylinders are measured to increase the statistic accuracy. As can be seen from the plot in Figure 4C, the lasing threshold has a minimum in the vicinity of $D=500 \mathrm{~nm}$, indicating an increase in the $Q$ factor of the mode for this diameter, which is in a good agreement with the theoretically obtained behavior for the supercavity condition.

\section{CONCLUSION}

In summary, using the concept of quasi-bound states in the continuum or supercavity modes, we have demonstrated lasing action at cryogenic temperatures in a single GaAs nanocylinder fabricated on a quartz substrate. The nanolaser has a size-towavelength ratio as low as 0.6 , which, to the best of our knowledge, is one of the lowest values demonstrated for purely semiconductor nanolasers so far. This became possible by engineering two resonances supported by the system, namely, the Mie resonance and the Fabry-Perot resonance, to achieve a quasi-bound state in the continuum, the so-called supercavity mode, via mutual destructive interference of the resonances in the far field at the targeted lasing wavelength. As compared to previous approaches, the present design does not need the particle to be suspended in air and does not rely on modes of high azimuthal orders, which increases the robustness of the device. We believe that this approach can further be brought to room-temperature operation by using higher gain materials or surface passivation techniques. GaAs is known to have a strong nonradiative recombination due to surface defects which significantly lowers its gain property. There are two ways to reduce the surface recombination: first, using a surface passivation by chemical method (passivating using sulfide compounds such as $\mathrm{P}_{2} \mathrm{~S}_{5},\left(\mathrm{NH}_{4}\right)_{2} \mathrm{~S}, \mathrm{Na}_{2} \mathrm{~S}$, etc.), and second, using a passivation layer like AlGaAs or multiquantum well (MQW) structure of AlGaAs/GaAs. Such MQW structure, which is widely used in high-efficient LEDs and lasers, is a good candidate for room-temperature operation for this type of laser if we can preserve its emitting property during the dryetching process. In the future, with the use of electrical pumping and radiation out-coupling strategies, the presented concept can be useful for smaller, Ohmic-loss-free nanolasers, with potential applications in photonic circuits and beyond.

\section{METHODS}

Numerical Simulations. Numerical simulations were performed using an FDTD commercial software (Lumerical FDTD Solutions). There are two types of numerical simulations carried out in this work: nanoparticle scattering simulations and $Q$ factor calculations. In nanoparticle scattering simulations, the cylinder was irradiated by a plane wave using total-field scattered-field (TFSF) source. This source separates the scattered field from the incident field, which allows us to compute scattering efficiency $Q_{\text {sca. }}$. The scattering efficiency was obtained using the following relation, ${ }^{47} Q_{\text {sca }}(\lambda)=W_{\text {sca }}(\lambda) \cdot\left(I_{s}(\lambda) \cdot D\right.$. $H)^{-1}$, where $\lambda$ is the wavelength of radiation, $W_{\text {sca }}$ is the power scattered through a box monitor (closed surface) surrounding the cylinder, $I_{\mathrm{s}}$ is the intensity of the source irradiating the nanoparticle, $D$ is the diameter of the cylinder, and $H$ is its height. Two types of plane waves were considered for excitation, namely, TE polarized $\left(E_{z}=0\right.$, $H_{z} \neq 0$, Figure $\left.1 \mathrm{~A}\right)$ and TM polarized $\left(H_{z}=0, E_{z} \neq 0\right)$, where $z$ is the axis of the cylinder and $E_{z}$ and $H_{z}$ are the projections of electric field and magnetic field onto the $z$ axis, respectively. From the scattering simulations, the scattering efficiency map $Q_{\text {sca }}(\lambda, D)$, shown in Figure $1 C$, was obtained. To compute the quasi-normal eigenmodes, and the corresponding $Q$ factors, one electric dipole was placed inside the dielectric cylinder to selectively excite the modes. To do so, the dipole was placed in the vicinity of the antinode of the electric field of the desired mode with its dipole moment parallel to the electric field at that point (as, for example, depicted in Figure 1B, for excitation of quasi-TE modes). Time-dependent electric and magnetic fields were recorded using nine pointlike time monitors, randomly placed inside the cylinder. After that, the spectrum was calculated for each monitor using fast Fourier transform (FFT). Then the spectra were averaged over the number of monitors obtaining the spectrum for $Q$ factor calculations. The resonant peaks in the spectrum were fit by a Lorentzian function to obtain $\Delta f_{i}$, fwhm for the $i$ th resonance. The $Q$ factor of the $i$ th resonance was calculated using $Q=f_{i} / \Delta f_{i}$ where $f_{i}$ is the frequency of the $i$ th resonance. The far-field $3 \mathrm{D}$ pattern of the modes was computed using a box monitor completely surrounding the cylinder. The far-field 2D pattern of the modes was obtained using a plane monitor (perpendicular to the $z$ axis) located above the cylinder.

In order to account for the real experimental conditions, additional numerical simulations and optimization were performed considering that the dielectric nanocylinder is placed on a quartz substrate and has a capping HSQ layer on top, as can be seen in the SEM image in the inset of Figure $2 \mathrm{~B}$. In those simulations, we assumed that the cylinder had a constant relative permittivity $\varepsilon=13$, that the HSQ cylinder on top had a height of $150 \mathrm{~nm}$ and a constant refractive index $n_{\mathrm{HSQ}}=$ 1.41 and that the substrate had a constant refractive index $n_{\text {substrate }}=$ 1.46.

Sample Nanofabrication. GaAs wafer with an epitaxial structure of i-GaAs $(500 \mathrm{~nm}) / \mathrm{AlAs}(100 \mathrm{~nm}) /$ undoped $\langle 100\rangle \mathrm{GaAs}(625 \mathrm{~nm})$ was purchased from Semiconductor Wafer, Inc., Taiwan. Epitaxial liftoff $^{38}$ technique was used to transfer the top, high quality GaAs film $(500 \mathrm{~nm})$ from the wafer to a quartz substrate. In the process, black wax (i.e., Apiezon $\mathrm{W}$ ) was applied on the top i-GaAs layer and thermally annealed at $90{ }^{\circ} \mathrm{C}$ for about $30-60 \mathrm{~min}$ to realize a slightly domed surface. After that, hydrofluoric acid (HF) solution ( $\sim 5 \mathrm{wt} \%$ in $\mathrm{H}_{2} \mathrm{O}$ ) was used to etch the sacrificial layer (AlAs) and GaAs epitaxial film was then lifted off. Direct bonding process was used to attach the film to the quartz substrate using a wafer bonder system (EVG 510). Inductively coupled plasma reactive ion etching (ICP RIE) tool (Oxford PlasmaLab System 100) was used to etch GaAs film to a desired thickness. Standard electron beam lithography procedure (Elionix ELS-7000 system) was used to fabricate nanocylinders mask using hydrogen silsesquioxane resist (HSQ Dow Corning XR-1541-006) along with ESpacer (E-spacer 300Z) to improve the conductivity during e-beam exposure. The unexposed resist was removed using $25 \%$ tetramethylammonium hydroxide (TMAH) developer. Finally, the patterned GaAs film was etched using ICP RIE. Nanocylinders were fabricated with a $20 \mu \mathrm{m}$ spacing. That period was chosen in such a way that each cylinder would be individually irradiated during subsequent optical measurements, thus acting as an isolated nanoparticle.

Optical Characterization. All optical measurements were performed in a microspectrometer setup which consists of an inverted microscope (Nikon Ti-U) and a spectrometer system (Andor SR303i spectrograph coupled with a $400 \times 1600$-pixel Electron Multiplying Charge-Coupled Device - Newton 971 EMCCD). For lasing and photoluminescence measurements, a Ti:sapphire femtosecond laser (Coherent, MIRA 900) was used. The pumping wavelength was 
tuned to $\sim 530 \mathrm{~nm}$ by using an optical parametric amplifier (Coherent OPA 9450, RegA). The pulse duration and repetition rate after OPA are $\sim 200 \mathrm{fs}$ and $20 \mathrm{kHz}$, respectively. The laser beam was focused on the nanocylinder using a 50X dark-field (DF) microscope objective (Nikon, CFI LU Plan DF, numerical aperture NA $=0.55$ ) resulting in a laser spot of $\sim 5 \mu \mathrm{m}$ in diameter. The dark-field scattering was measured in a backward scattering configuration using the same microscope objective with white light excitation from a halogen lamp. Transmission of GaAs thin film was measured using another halogen lamp excitation. The absorption was calculated by the formula $A=2-$ $\log _{10}(\% T)$. All spectrally resolved measurements were carried out with 150 grooves $/ \mathrm{mm}, 800 \mathrm{~nm}$ blading grating and the spectrograph slit width of $100 \mu \mathrm{m}$ resulting in a spectral resolution of $\sim 1 \mathrm{~nm}$. All measurements were performed at cryogenic temperatures. For that, the sample was placed onto the coldfinger of a cryostat (Janis, ST500 ). The chamber was vacuumed to $3 \times 10^{-3}$ Torr by an oil pump before being cooled to $77 \mathrm{~K}$ using liquid nitrogen.

Autocorrelation Measurements. Autocorrelation measurements were carried out using a Hanbury-Brown-Twiss setup. The emission signal in our microscopic lasing measurement setup was routed into a multimode fiber (Thorlabs GIF625, $62.5 \mu \mathrm{m}$ core). The signal was then sent to a 50:50 optical fiber beamsplitter with each node coupled with a low-jitter avalanche photodiodes (Micro Photon Devices, PDM series with a timing jitter of $35 \mathrm{ps}$ fwhm). Coincident clicks on the two photodiodes were recorded using a time-tagging device (qutools, QuTAU, timing resolution $81 \mathrm{ps}$ ).

\section{ASSOCIATED CONTENT}

\section{SI Supporting Information}

The Supporting Information is available free of charge at https://pubs.acs.org/doi/10.1021/acsnano.0c02730.

Measured absorption of the GaAs film (Figure S1), onedimensional spectra, obtained in the scattering and lasing experiments (Figure S2), and the $Q$ factor and near-field analysis of the modes $\mathrm{TE}_{3}$ and $\mathrm{TM}_{410}$ (Figure S3) (PDF)

\section{AUTHOR INFORMATION}

\section{Corresponding Author}

Arseniy I. Kuznetsov - Institute of Materials Research and Engineering, $A$ *STAR (Agency for Science, Technology and Research), Innovis, Singapore 138634; 이이.org/00000002-7622-8939; Email: arseniy_kuznetsov@imre.astar.edu.sg

\section{Authors}

Vasilii Mylnikov - Institute of Materials Research and Engineering, A*STAR (Agency for Science, Technology and Research), Innovis, Singapore 138634; LUMINOUS! Center of Excellence for Semiconductor Lighting and Displays, The Photonics Institute, School of Electrical and Electronic Engineering, School of Physical and Mathematical Sciences, Nanyang Technological University, Singapore 639798

Son Tung Ha - Institute of Materials Research and Engineering, A*STAR (Agency for Science, Technology and Research), Innovis, Singapore 138634; 이이.org/0000-0002-54758365

Zhenying Pan - Institute of Materials Research and Engineering, A*STAR (Agency for Science, Technology and Research), Innovis, Singapore 138634

Vytautas Valuckas - Institute of Materials Research and Engineering, A*STAR (Agency for Science, Technology and Research), Innovis, Singapore 138634

Ramón Paniagua-Domínguez - Institute of Materials Research and Engineering, A*STAR (Agency for Science, Technology and
Research), Innovis, Singapore 138634; 이 orcid.org/00000001-7836-681X

Hilmi Volkan Demir - LUMINOUS! Center of Excellence for Semiconductor Lighting and Displays, The Photonics Institute, School of Electrical and Electronic Engineering, School of Physical and Mathematical Sciences, Nanyang Technological University, Singapore 639798; Bilkent University UNAM Institute of Nanotechnology and Materials Science, Department of Electrical and Electronic Engineering, Department of Physics, Bilkent University, Ankara 06800, Turkey; 이이이.org/00000003-1793-112X

Complete contact information is available at:

https://pubs.acs.org/10.1021/acsnano.0c02730

\section{Author Contributions}

R.P.-D. and A.I.K. conceived the idea. V.M. performed numerical simulations. Z.P. performed epitaxial lift-off of the GaAs film, its bonding to the quartz substrate and etching. V.M. performed etching, electron beam lithography and etching after EBL. V.V. performed SEM measurements. S.T.H. constructed the optical setup, performed all optical measurements and their data processing. All authors discussed the results. V.M. wrote the first draft of the manuscript and all authors worked on it. R.P.-D., H.V.D., and A.I.K. coordinated and supervised the work.

\section{Notes}

The authors declare no competing financial interest.

\section{ACKNOWLEDGMENTS}

The authors acknowledge Leonid Krivitsky (IMRE, A*STAR) and Victor Leong Xu Heng (IMRE, A*STAR) for processing autocorrelation function measurements data. The authors acknowledge the financial support from A*STAR: SERC Pharos program, Singapore (Grant No. 15273 00025) and Singapore International Graduate Award.

\section{REFERENCES}

(1) Kuznetsov, A. I.; Miroshnichenko, A. E.; Brongersma, M. L.; Kivshar, Y. S.; Luk'yanchuk, B. Optically Resonant Dielectric Nanostructures. Science 2016, 354, aag2472.

(2) Paniagua-Domínguez, R.; Ha, S. T.; Kuznetsov, A. I. Active and Tunable Nanophotonics with Dielectric Nanoantennas. Proc. IEEE 2020, 108, 749-771.

(3) Krasnok, A.; Caldarola, M.; Bonod, N.; Alù, A. Spectroscopy and Biosensing with Optically Resonant Dielectric Nanostructures. Adv. Opt. Mater. 2018, 6, 1701094.

(4) Regmi, R.; Berthelot, J.; Winkler, P. M.; Mivelle, M.; Proust, J.; Bedu, F.; Ozerov, I.; Begou, T.; Lumeau, J.; Rigneault, H.; GarcíaParajó, M. F.; Bidault, S.; Wenger, J.; Bonod, N. All-Dielectric Silicon Nanogap Antennas to Enhance the Fluorescence of Single Molecules. Nano Lett. 2016, 16, 5143-5151.

(5) Trofymchuk, K.; Reisch, A.; Didier, P.; Fras, F.; Gilliot, P.; Mely, Y.; Klymchenko, A. S. Giant Light-Harvesting Nanoantenna for Single-Molecule Detection in Ambient Light. Nat. Photonics 2017, 11, 657-663.

(6) Caldarola, M.; Albella, P.; Cortés, E.; Rahmani, M.; Roschuk, T.; Grinblat, G.; Oulton, R. F.; Bragas, A. V.; Maier, S. A. Non-Plasmonic Nanoantennas for Surface Enhanced Spectroscopies with Ultra-Low Heat Conversion. Nat. Commun. 2015, 6, 7915.

(7) Staude, I.; Khardikov, V. V.; Fofang, N. T.; Liu, S.; Decker, M.; Neshev, D. N.; Luk, T. S.; Brener, I.; Kivshar, Y. S. Shaping Photoluminescence Spectra with Magnetoelectric Resonances in AllDielectric Nanoparticles. ACS Photonics 2015, 2, 172-177.

(8) Bouchet, D.; Mivelle, M.; Proust, J.; Gallas, B.; Ozerov, I.; Garcia-Parajo, M. F.; Gulinatti, A.; Rech, I.; De Wilde, Y.; Bonod, N.; 
Krachmalnicoff, V.; Bidault, S. Enhancement and Inhibition of Spontaneous Photon Emission by Resonant Silicon Nanoantennas. Phys. Rev. Appl. 2016, 6, 064016.

(9) Rodriguez, I.; Shi, L.; Lu, X.; Korgel, B. A.; Alvarez-Puebla, R. A.; Meseguer, F. Silicon Nanoparticles as Raman Scattering Enhancers. Nanoscale 2014, 6, 5666-5670.

(10) Dmitriev, P. A.; Baranov, D. G.; Milichko, V. A.; Makarov, S. V.; Mukhin, I. S.; Samusev, A. K.; Krasnok, A. E.; Belov, P. A.; Kivshar, Y. S. Resonant Raman Scattering from Silicon Nanoparticles Enhanced by Magnetic Response. Nanoscale 2016, 8, 9721-9726.

(11) Han, X. X.; Ji, W.; Zhao, B.; Ozaki, Y. SemiconductorEnhanced Raman Scattering: Active Nanomaterials and Applications. Nanoscale 2017, 9, 4847-4861.

(12) Zalogina, A. S.; Savelev, R. S.; Ushakova, E. V.; Zograf, G. P.; Komissarenko, F. E.; Milichko, V. A.; Makarov, S. V.; Zuev, D. A.; Shadrivov, I. V. Purcell Effect in Active Diamond Nanoantennas. Nanoscale 2018, 10, 8721-8727.

(13) Rutckaia, V.; Heyroth, F.; Novikov, A.; Shaleev, M.; Petrov, M.; Schilling, J. Quantum Dot Emission Driven by Mie Resonances in Silicon Nanostructures. Nano Lett. 2017, 17, 6886-6892.

(14) Tiguntseva, E. Y.; Zograf, G. P.; Komissarenko, F. E.; Zuev, D. A.; Zakhidov, A. A.; Makarov, S. V.; Kivshar, Y. S. Light-Emitting Halide Perovskite Nanoantennas. Nano Lett. 2018, 18, 1185-1190.

(15) Liu, S.; Vaskin, A.; Addamane, S.; Leung, B.; Tsai, M. C.; Yang, Y.; Vabishchevich, P. P.; Keeler, G. A.; Wang, G.; He, X.; Kim, Y.; Hartmann, N. F.; Htoon, H.; Doorn, S. K.; Zilk, M.; Pertsch, T.; Balakrishnan, G.; Sinclair, M. B.; Staude, I.; Brener, I. Light-Emitting Metasurfaces: Simultaneous Control of Spontaneous Emission and Far-Field Radiation. Nano Lett. 2018, 18, 6906-6914.

(16) Ha, S. T.; Fu, Y. H.; Emani, N. K.; Pan, Z.; Bakker, R. M.; Paniagua-Domínguez, R.; Kuznetsov, A. I. Directional Lasing in Resonant Semiconductor Nanoantenna Arrays. Nat. Nanotechnol. 2018, 13, 1042-1047.

(17) Kodigala, A.; Lepetit, T.; Gu, Q.; Bahari, B.; Fainman, Y.; Kanté, B. Lasing Action from Photonic Bound States in Continuum. Nature 2017, 541, 196-199.

(18) Wang, Z.; Meng, X.; Kildishev, A. V.; Boltasseva, A.; Shalaev, V. M. Nanolasers Enabled by Metallic Nanoparticles: From Spasers to Random Lasers. Laser Photonics Rev. 2017, 11, 1700212.

(19) Zhang, Z.; Yang, L.; Liu, V.; Hong, T.; Vahala, K.; Scherer, A. Visible Submicron Microdisk Lasers. Appl. Phys. Lett. 2007, 90, 111119.

(20) Song, Q.; Cao, H.; Ho, S. T.; Solomon, G. S. Near-IR Subwavelength Microdisk Lasers. Appl. Phys. Lett. 2009, 94, 061109.

(21) Tiguntseva, E. Y.; Koshelev, K. L.; Furasova, A. D.; Mikhailovskii, V. Y.; Ushakova, E. V.; Baranov, D. G.; Shegai, T. O.; Zakhidov, A. A.; Kivshar, Y. S.; Makarov, S. V. Single-Particle MieResonant All-Dielectric Nanolasers. arXiv 2019, 1905.08646 https:// arxiv.org/abs/1905.08646 (accessed 2019-05-25).

(22) Hsu, C. W.; Zhen, B.; Stone, A. D.; Joannopoulos, J. D.; Soljačić, M. Bound States in the Continuum. Nat. Rev. Mater. 2016, 1 , 16048 .

(23) Rybin, M.; Kivshar, Y. Supercavity Lasing. Nature 2017, 541, 164-165.

(24) Koshelev, K.; Favraud, G.; Bogdanov, A.; Kivshar, Y.; Fratalocchi, A. Nonradiating Photonics with Resonant Dielectric Nanostructures. Nanophotonics 2019, 8, 725-745.

(25) Tittl, A.; Leitis, A.; Liu, M.; Yesilkoy, F.; Choi, D.-Y.; Neshev, D. N.; Kivshar, Y. S.; Altug, H. Imaging-Based Molecular Barcoding with Pixelated Dielectric Metasurfaces. Science 2018, 360, 1105-1109.

(26) Yesilkoy, F.; Arvelo, E. R.; Jahani, Y.; Liu, M.; Tittl, A.; Cevher, V.; Kivshar, Y.; Altug, H. Ultrasensitive Hyperspectral Imaging and Biodetection Enabled by Dielectric Metasurfaces. Nat. Photonics 2019, 13, 390-396.

(27) Carletti, L.; Koshelev, K.; De Angelis, C.; Kivshar, Y. Giant Nonlinear Response at the Nanoscale Driven by Bound States in the Continuum. Phys. Rev. Lett. 2018, 121, 033903.

(28) Koshelev, K.; Bogdanov, A.; Kivshar, Y. Meta-Optics and Bound States in the Continuum. Sci. Bull. 2019, 64, 836-842.
(29) Carletti, L.; Kruk, S. S.; Bogdanov, A. A.; De Angelis, C.; Kivshar, Y. High-Harmonic Generation at the Nanoscale Boosted by Bound States in the Continuum. Phys. Rev. Res. 2019, 1, 023016.

(30) Koshelev, K.; Kruk, S.; Melik-Gaykazyan, E.; Choi, J.-H.; Bogdanov, A.; Park, H.-G.; Kivshar, Y. Subwavelength Dielectric Resonators for Nonlinear Nanophotonics. Science 2020, 367, 288292.

(31) Volkovskaya, I. I.; Xu, L.; Huang, L.; Smirnov, A. I.; Miroshnichenko, A. Multipolar Second-Harmonic Generation from High-Q Quasi-BIC States in Nanoresonators. arXiv 2020, 2001.04318, https://arxiv.org/abs/2001.04318 (accessed 2020-0326).

(32) Henry, C. H.; Kazarinov, R. F.; Logan, R. A.; Yen, R. Observation of Destructive Interference in the Radiation Loss of Second-Order Distributed Feedback Lasers. IEEE J. Quantum Electron. 1985, 21, 151-154.

(33) Silveirinha, M. G. Trapping Light in Open Plasmonic Nanostructures. Phys. Rev. A: At., Mol., Opt. Phys. 2014, 89, 023813.

(34) Monticone, F.; Alù, A. Embedded Photonic Eigenvalues in 3D Nanostructures. Phys. Rev. Lett. 2014, 112, 213903.

(35) Rybin, M. V.; Koshelev, K. L.; Sadrieva, Z. F.; Samusev, K. B.; Bogdanov, A. A.; Limonov, M. F.; Kivshar, Y. S. High-Q Supercavity Modes in Subwavelength Dielectric Resonators. Phys. Rev. Lett. 2017, $119,243901$.

(36) Chen, W.; Chen, Y.; Liu, W. Multipolar Conversion Induced Subwavelength High-Q Kerker Supermodes with Unidirectional Radiations. Laser Photonics Rev. 2019, 13, 1900067.

(37) Bogdanov, A. A.; Koshelev, K. L.; Kapitanova, P. V.; Rybin, M. V.; Gladyshev, S. A.; Sadrieva, Z. F.; Samusev, K. B.; Kivshar, Y. S.; Limonov, M. F. Bound States in the Continuum and Fano Resonances in the Strong Mode Coupling Regime. Adv. Photon. 2019, 1, 016001

(38) Yablonovitch, E.; Gmitter, T.; Harbison, J. P.; Bhat, R. Extreme Selectivity in the Lift-Off of Epitaxial GaAs Films. Appl. Phys. Lett. $1987,51,2222-2224$.

(39) Hessel, C. M.; Henderson, E. J.; Veinot, J. G. C. Hydrogen Silsesquioxane: A Molecular Precursor for Nanocrystalline $\mathrm{Si}-\mathrm{SiO}_{2}$ Composites and Freestanding Hydride-Surface-Terminated Silicon Nanoparticles. Chem. Mater. 2006, 18, 6139-6146.

(40) Zhou, W.-J.; Zheng, Y.-X.; Zhang, C.; Ma, X.-F.; Li, D.-H.; Ma, L.; Hu, F.; Yang, S.-D.; Yang, L.; Gao, M.-Y.; Lu, M.; Zhang, R.-J.; Wang, S.-Y.; Chen, L.-Y. Optical Properties of High Photoluminescence Silicon Nanocrystals Embedded in $\mathrm{SiO}_{2}$ Matrices Obtained By Annealing Hydrogen Silsesquioxane. Opt. Mater. 2018, 84, 874-878.

(41) Choi, S.; Word, M. J.; Kumar, V.; Adesida, I. Comparative Study of Thermally Cured and Electron-Beam-Exposed Hydrogen Silsesquioxane Resists. J. Vac. Sci. Technol., B: Microelectron. Nanometer Struct.-Process., Meas., Phenom. 2008, 26, 1654-1659.

(42) Wang, D.-C.; Zhang, C.; Zeng, P.; Zhou, W.-J.; Ma, L.; Wang, H.-T.; Zhou, Z.-Q.; Hu, F.; Zhang, S.-Y.; Lu, M.; Wu, X. An AllSilicon Laser Based on Silicon Nanocrystals with High Optical Gains. Sci. Bull. 2018, 63, 75-77.

(43) Numai, T. Fundamentals of Semiconductor Lasers; Springer: Tokyo, 2015.

(44) Saxena, D.; Mokkapati, S.; Parkinson, P.; Jiang, N.; Gao, Q.; Tan, H. H.; Jagadish, C. Optically Pumped Room-Temperature GaAs Nanowire Lasers. Nat. Photonics 2013, 7, 963-968.

(45) Chow, W. W.; Jahnke, F.; Gies, C. Emission Properties of Nanolasers during the Transition to Lasing. Light Sci. Appl. 2014, 3, No. e201.

(46) Pan, S. H.; Gu, Q.; Amili, A. E.; Vallini, F.; Fainman, Y. Dynamic Hysteresis in a Coherent High- $\beta$ Nanolaser. Optica 2016, 3, $1260-1265$

(47) Bohren, C. F.; Huffman, D. R. Absorption and Scattering of Light by Small Particles; John Wiley \& Sons Inc: New York, 1983. 